\title{
Division Algebras with Left Algebraic Commutators
}

\author{
M. Aaghabali ${ }^{1}$ (D) S. Akbari' ${ }^{2}$ M. H. Bien ${ }^{3}$
}

Received: 24 January 2017 / Accepted: 19 September 2017 / Published online: 3 October 2017

(C) The Author(s) 2017. This article is an open access publication

\begin{abstract}
Let $D$ be a division algebra with center $F$ and $K$ a (not necessarily central) subfield of $D$. An element $a \in D$ is called left algebraic (resp. right algebraic) over $K$, if there exists a non-zero left polynomial $a_{0}+a_{1} x+\cdots+a_{n} x^{n}$ (resp. right polynomial $a_{0}+x a_{1}+\cdots+x^{n} a_{n}$ ) over $K$ such that $a_{0}+a_{1} a+\cdots+a_{n} a^{n}=0$ (resp. $\left.a_{0}+a a_{1}+\cdots+a^{n} a_{n}\right)$. Bell et al. proved that every division algebra whose elements are left (right) algebraic of bounded degree over a (not necessarily central) subfield must be centrally finite. In this paper we generalize this result and prove that every division algebra whose all multiplicative commutators are left (right) algebraic of bounded degree over a (not necessarily central) subfield must be centrally finite provided that the center of division algebra is infinite. Also, we show that every division algebra whose multiplicative group of commutators is left (right) algebraic of bounded degree over a (not necessarily central) subfield must be centrally finite. Among other results we present similar result regarding additive commutators under certain conditions.
\end{abstract}

Presented by Michel Van den Bergh.

Dedicated to Professor Jeffrey Bergen

M. Aaghabali

mehdi.aaghabali@ed.ac.uk

S. Akbari

s_akbari@sharif.edu

M.H. Bien

maihoangbien012@yahoo.com

1 School of Mathematics, The University of Edinburgh, James Clerk Maxwell Building, The Kings Buildings, Mayfield Road EH9 3JZ, Edinburgh, UK

2 Department of Mathematical Sciences, Sharif University of Technology, P.O. Box 11155-9415, Tehran, Iran

3 Faculty of Mathematics and Computer Science, University of Science, VNU-HCM, 227 Nguyen Van Cu Str., Dist. 5, HCM-City, Vietnam 
Keywords Division algebra $\cdot$ Commutators $\cdot$ Laurent polynomial identity $\cdot$ Maximal subfield · Left algebraic

Mathematics Subject Classification (2010) 05 A05 $\cdot$ 05E15 $\cdot 16$ K20 $\cdot$ 17A35

\section{Introduction}

A theorem due to Jacobson [7] says that every algebraic division algebra of bounded degree over its center, is centrally finite. Akbari et al. in [10] generalized this theorem and proved that the statement holds if one assumes that $D$ is a division algebra whose multiplicative group of commutators is algebraic of bounded degree over the center. Recently, Bell et al. in [4] using a technique based on combinatorics of words have provided a generalization of Jacobson Theorem from other aspects of view. They proved that every division algebra whose elements are algebraic of bounded degree over a (not necessarily central) subfield, must be centrally finite. More precisely, they proved that if $D$ is a division algebra with center $F$ such that its elements are left algebraic of bounded degree $n$ over a subfield $K$, then $\operatorname{dim}_{F} D \leq n^{2}$.

For any centrally finite division algebra $D$ over $F$, by Kothe's Theorem it is known that there exists a maximal subfield $K$ of $D$ such that the extension of fields $K / F$ is separable [8, p. 244]. In [10], authors proved that if $K / F$ is a finite separable extension of fields in $D$, then there exists an element $d \in D^{\prime}$, the multiplicative group of commutators of $D^{*}$, such that $K=F(d)$. Now, one may ask: whether $F(d)$ is a maximal subfield of $D$ for some $d$ in additive or multiplicative groups of commutators. Chebotar et al. in [5] answered the question in affirmative in the case of centrally finite division algebras. In this note we give a new proof for these questions. Also, we concern with a generalization of a theorem due to Akbari et al. [10] and Bell et al. [4]. In fact we prove that a division algebra whose all multiplicative commutators are left (right) algebraic of bounded degree over a (not necessarily central) subfield is centrally finite, provided that has infinite center. As another result we show that if $D$ is a division algebra with center $F$ whose multiplicative group of commutators is left (right) algebraic of bounded degree $n$ over a (not necessarily central) subfield, then $\operatorname{dim}_{F} D \leq n^{2}$. Among other results we prove that if $D$ is a division algebra with infinite center $F$ and non-central subfield $K$ with $a \in K \backslash F$ algebraic over $F$ such that for every $x \in D, a x-x a$ is left (right) algebraic over subfield $K$ of bounded degree $n$, then $D$ is centrally finite.

\section{Preliminary}

Let $K$ be a subdivision algebra of $D$ with center $F$. An element $a \in D$ is called left algebraic (resp. right algebraic) over $K$, if there exists a non-zero left polynomial $a_{0}+a_{1} x+\cdots+a_{n} x^{n}$ (resp. right polynomial $a_{0}+x a_{1}+\cdots+x^{n} a_{n}$ ) over $K$ such that $a_{0}+a_{1} a+\cdots+a_{n} a^{n}=0$ (resp. $a_{0}+a a_{1}+\cdots+a^{n} a_{n}$ ). In other words, $a$ is left (resp. right) algebraic over $K$ if the set $\left\{1, a, a^{2}, \ldots\right\}$ is a dependent set of left (resp. right) vector space $D$ over $K$. In this case, assume that $n$ is the smallest degree of left polynomials $f(x)$ (resp. right polynomial) such that $f(a)=0$. We denote by $\operatorname{deg}_{K}(a)=n$ (resp. $\left.K \operatorname{deg}(a)=n\right)$ the left degree (resp. right degree) of $a$ over $K$. If $K=F$ is the center, then the concept of left and right algebraic coincides. It is easy to see that if $a$ is left algebraic over $K$, then the left degree of $a$ over $K$ is the smallest integer $n$ such that $\left\{1, a, \ldots, a^{n}\right\}$ is left dependent over $K$. If $a \in D$, then 
$F(a)$ denotes the subfield of $D$ generated by $F$ and $\{a\}$. If $A$ is a subset of $D$, then $K(A)$ denotes the subdivision algebra generated by $K \cup A$. Also, for a subset $A$ of $D$, we use $K[A]$ to denote the subalgebra generated by $K \cup A$. When $A=\{a\}$, then we use $K[a]$ to denote subalgebra generated by $K \cup\{a\}$. If $A$ is a subset of $D$ we use $A^{*}$ to denote $A \backslash\{0\}$. A subfield $K$ of $D$ is called a maximal subfield if $K$ is its own centralizer in $D^{*}$. We denote by $\operatorname{dim}_{F} D, Z(D)$ and $\operatorname{char}(D)$ the dimension of $D$ over $F$, center of $D$ and characteristic of $D$, respectively. If $\operatorname{dim}_{F} D=n^{2}$, then $n$ is called the degree of division algebra $D$. By $M_{n}(K)$ and $G L_{n}(K)$ we mean all square matrices and all invertible matrices of order $n$ with entries from $K$, respectively.

Before stating our results we need to recall some theorems. The first one is a generalization of celebrated theorem due to Brauer, Cartan and Hua.

Theorem 1 [6] Let $D$ be a division algebra with center $F$ and $H$ a proper subdivision algebra of $D$ such that $H$ is invariant under a subnormal non-central subgroup $G$ in $D^{*}$, that is $\mathrm{gHg}^{-1} \subseteq H$ for every $g \in G^{*}$. Then $H \subseteq F$.

Theorem 2 [8, p. 242] If $D$ is a division algebra of degree $n$ over a field $F$ and $K$ is a subfield of $D$ containing $F$, then $\operatorname{dim}_{F} K \leq n$. The quality holds if and only if $K$ is a maximal subfield of $D$. Conversely, if $K$ is a maximal subfield of a division algebra $D$ such that $\operatorname{dim}_{F} K=n<\infty$, then $D$ is of degree $n$ over $F$.

Let $X$ be a set of non-commutating indeterminates and $F$ be an arbitrary field. The Laurent polynomial ring $F\left[X, X^{-1}\right]$ is the ring of all finite sums of form

$$
\alpha_{n} x_{n_{1}}^{m_{1}} x_{n_{2}}^{m_{2}} \ldots x_{n_{t}}^{m_{t}},
$$

where $\alpha_{n} \in F, x_{n_{i}} \in X$ and $m_{i} \in\{1,-1\}$. Every element of $F\left[X, X^{-1}\right]$ is called a Laurent polynomial. A Laurent polynomial $f \in F\left[X, X^{-1}\right]$ is called a Laurent polynomial identity of a ring $R$ if it vanishes on all permissible substitutions from $R$. In this case, we say that $R$ satisfies the Laurent polynomial identity $f=0$. We consider the following example which is important in this paper. Given a positive integer $n$, and $n+1$ non-commutating indeterminates $y_{0}, y_{1}, \ldots, y_{n}$, define

$$
g_{n}\left(y_{0}, y_{1}, y_{2}, \ldots, y_{n}\right)=\sum_{\delta \in S_{n+1}} \operatorname{sign}(\delta) y_{0}^{\delta(0)} y_{1} y_{0}^{\delta(1)} y_{2} y_{0}^{\delta(2)} \ldots y_{n} y_{0}^{\delta(n)},
$$

where $S_{n+1}$ is the symmetric group over $\{0,1, \ldots, n\}$ and $\operatorname{sign}(\delta)$ is the sign of permutation $\delta$. This is a Laurent polynomial over any field and it is defined in [3] to connect an algebraic element of degree $n$ and a polynomial of $n+1$ indeterminates.

Lemma 3 Let $F$ be a field and A be a central simple algebra over F. For any element $a \in A$, the following conditions are equivalent:

1. The element a is algebraic over $F$ of degree less than $n$.

2. $g_{n}\left(a, r_{1}, \ldots, r_{n}\right)=0$ for any $r_{1}, \ldots, r_{n} \in A$.

Proof This is a corollary of [3, Corollary 2.3.8].

In particular, a centrally finite division algebra of degree $m$ satisfies the identity $g_{m}$ since whose elements are algebraic over the center of degree $m$. In other words, $g_{m}$ is a Laurent polynomial identity of any centrally finite division algebra of degree $m$. 
Let $\mathcal{I}(A)$ denote the set of all Laurent polynomial identities of the centrally finite division algebra $A$. The following theorem gives us a relation between the set of all Laurent polynomial identities of central simple algebras.

Theorem 4 Let $F$ be an infinite field and A be a central simple algebra of degree $n$ over $F$. Assume that $L$ is an extension field of $F$. Then

$$
\mathcal{I}(A)=\mathcal{I}\left(M_{n}(F)\right)=\mathcal{I}\left(M_{n}(L)\right) .
$$

Proof This result is just a corollary of [2, Theorem 11].

In what follows we show that every centrally finite division algebra has a maximal subfield that is obtained by adjunction of a multiplicative or additive commutator to the center of division algebra. First we need to prove the following lemma.

Lemma 5 Let $K$ be a field and $n \geq 2$ be an integer.

(i) There exist two matrices $A, B \in M_{n}(K)$ such that the commutator $A B A^{-1} B^{-1}$ is an algebraic element of degree $n$ over $K$.

(ii) There exist two matrices $A, B \in M_{n}(K)$ such that $A B-B A$ is an algebraic element of degree $n$ over $K$.

Proof (i) It is well-known that every matrix of determinant one is a single multiplicative commutator [12]. Now, consider $n \times n$-matrix $T=\left(t_{i j}\right)$, where

$$
t_{i j}= \begin{cases}1 & , j=i \text { or } i+1 \\ 0 & , \text { otherwise }\end{cases}
$$

Then $T$ has determinant one and it is algebraic of degree $n$. This completes the proof of part $(i)$.

(ii) It is well-known that every matrix with trace zero is a single additive commutator [1]. Now, consider $n \times n$-matrix $T=\left(t_{i j}\right)$, where

$$
t_{i j}= \begin{cases}1 & , j=i+1 \\ 0 & , \text { otherwise }\end{cases}
$$

Then $T$ has trace zero and it is algebraic of degree $n$. This completes the proof.

Theorem 6 Let $D$ be a centrally finite division algebra over a field $F$. There exist $x, y \in D^{*}$ such that $F\left(x y x^{-1} y^{-1}\right)$ is a maximal subfield of $D$.

Proof If $F$ is finite, then $D$ is also finite, so there is nothing to prove. Suppose that $F$ is infinite and $D$ is of degree $n$ over $F$. By Theorem 2, it suffices to show that there exist $x, y \in$ $D^{*}$ such that $\operatorname{dim}_{F} F\left(x y x^{-1} y^{-1}\right) \geq n$. Indeed, put $\ell=\max \left\{\operatorname{dim}_{F} F\left(x y x^{-1} y^{-1}\right) \mid x, y \in\right.$ $\left.D^{*}\right\}$. Applying Lemma 3 we find that $g_{\ell}\left(r s r^{-1} s^{-1}, r_{1}, \ldots, r_{\ell}\right)=0$, for any $r_{1}, \ldots, r_{\ell} \in D$ and $r, s \in D^{*}$. In other words, $g_{\ell}\left(x y x^{-1} y^{-1}, y_{1}, \ldots, y_{\ell}\right)$ is a Laurent polynomial identity of $D$. Hence, by Theorem 4 it is also a Laurent polynomial identity of $M_{n}(F)$. This yields that $g_{\ell}\left(X Y X^{-1} Y^{-1}, X_{1}, \ldots, X_{\ell}\right)=0$, for all matrices $X, Y \in G L_{n}(F)$ and $X_{i} \in M_{n}(F)$. By the first part of the previous lemma one can find matrices $A$ and $B$ in $G L_{n}(F)$ such that 
$A B A^{-1} B^{-1}$ is algebraic of degree $n$ over $F$. Hence, by Lemma 3 for any $X_{i} \in M_{n}(F)$ we have

$$
g_{n}\left(A B A^{-1} B^{-1}, X_{1}, \ldots, X_{n}\right)=g_{\ell}\left(A B A^{-1} B^{-1}, X_{1}, \ldots, X_{\ell}\right)=0 .
$$

Now, maximality of $\ell$ yields that $n \leq \ell$. This completes the proof.

Theorem 7 Let $D$ be a centrally finite division algebra with center $F$. There exist $x, y \in D$ such that $F(x y-y x)$ is a maximal subfield of $D$.

Proof If $F$ is finite then $D$ is also finite, so that there is nothing to prove. Suppose that $F$ is infinite and $D$ is of degree $n$. By Theorem 2, it suffices to show that there exist $x, y \in D$ such that $\operatorname{dim}_{F} F(x y-y x) \geq n$. Indeed, put $\ell=\max \left\{\operatorname{dim}_{F} F(x y-y x) \mid\right.$ $x, y \in D\}$. By Lemma $3, g_{\ell}\left(r s-s r, r_{1}, \ldots, r_{\ell}\right)=0$ for every $s, r, r_{1}, \ldots, r_{\ell} \in D$. It follows $g_{\ell}\left(X Y-Y X, X_{1}, \ldots, X_{\ell}\right)$ is a Laurent polynomial identity of $D$. From Theorem 4, $g_{\ell}\left(X Y-Y X, X_{1}, \ldots, X_{\ell}\right)$ is also a Laurent polynomial identity of $M_{n}(F)$. By the second part of the pervious lemma there exist $A, B \in M_{n}(F)$ such that $A B-B A$ is algebraic of degree $n$ over $F$ and one has $g_{\ell}\left(A B-B A, A_{1}, \ldots, A_{\ell}\right)=0$ for every $A_{i} \in M_{n}(F)$. Therefore, by Lemma 3, for any $X_{i} \in M_{n}(F)$ we have

$$
g_{n}\left(A B-B A, X_{1}, \ldots, X_{n}\right)=g_{\ell}\left(A B-B A, X_{1}, \ldots, X_{\ell}\right)=0 .
$$

Now, maximality of $\ell$ yields that $n \leq \ell$. This completes the proof.

\section{Combinatorics of Words}

The theory of identities gives us a technique to study algebraic elements of an algebra $R$ of bounded degree over a base field $F$. However, the base field in results are assumed to be contained in the center of $R$. The idea of [4] provides another technique to work on algebraic elements of bounded degree over any field. It is the theory of combinatorics on words.

Before stating our next result we need to remind the following theorems.

Theorem 8 [11, p. 156] Let $D$ be a division algebra and $K$ be a subfield of $D$. For $a \in D$, if $\operatorname{dim}_{K} K[a] \geq n$, then for any distinct elements $\alpha_{1}, \ldots, \alpha_{n} \in Z(D),\left(a-\alpha_{1}\right)^{-1}, \ldots,(a-$ $\left.\alpha_{n}\right)^{-1}$ are linearly independent.

Theorem 9 [4] Let $D$ be a division algebra with center $F$ whose elements are left algebraic of bounded degree $n$ over (not necessarily central) subfield $K$. Then $\operatorname{dim}_{F} D \leq n^{2}$.

The following lemma is crucial in next theorems.

Lemma 10 Let $D$ be a division algebra with infinite center $F$ and let $K$ be a subfield (not necessarily central) of $D$. If $a$ and $b$ are elements of $D^{*}$ such that for every central element $r, a(b+r)$ is left algebraic over $K$ of bounded degree $n$, then $\operatorname{dim}_{K} K[b] \leq 3^{2 n-1}+1$.

Proof Since $a(b+r)$ is left algebraic over $K$ of bounded degree $n$, thus we have

$$
\alpha_{m}(a(b+r))^{m-1} a(b+r)+\cdots+\alpha_{1} a(b+r)=-\alpha_{0},
$$

where $m \leq n, m$ is minimal and $a_{i} \in K$ for $0 \leq i \leq m$. Consequently there exists $h(x, y) \in$ $K[x, y]$ such that $h(a, b)(b+r)=1$. Now, consider all words of length at most $2 n-1$ consisting of two letters $a, b$ and let $W$ be the vector space generated by such words over 
$K$. Clearly, $\operatorname{dim}_{K} W \leq 3^{2 n-1}=k$ and for every $r \in F,(b+r)^{-1}=h(a, b) \in W$. Since $F$ is infinite, there are central elements $r_{1}, \ldots, r_{k+1}$ such that $\left(b+r_{1}\right)^{-1}, \ldots,\left(b+r_{k+1}\right)^{-1}$ are linearly dependent over $K$. Using Theorem 8, we find that $\operatorname{dim}_{K} K[b] \leq k+1$. This completes the proof.

Theorem 11 Let $D$ be a division algebra with infinite center $F$ and let $K$ be a subfield (not necessarily central) of $D$. Assume that there exists non-central element $a \in D$ such that for every $x \in D^{*}, x a x^{-1} a^{-1}$ is left algebraic over $K$ of bounded degree $n$. Then $D$ must be centrally finite.

Proof Consider $c \notin C_{D}(a)$. Hence for every $t \in F$ we have

$$
0 \neq b=a c-c a=a(c+t)-(c+t) a=a(c+t)\left(1-(c+t)^{-1} a^{-1}(c+t) a\right) .
$$

Put $u=b^{-1} a$ and observe that $u(c+t)$ is left algebraic over $K$ of bounded degree $n$. Hence, previous lemma implies that $\operatorname{dim}_{K} K[c] \leq 3^{2 n-1}+1=n^{\prime}$. In short, we could show that all elements of $D$ which are outside of $C_{D}(a)$ are left algebraic over $K$ of bounded degree $n^{\prime}$. Let $x \in C_{D}(a)$ and $z \notin C_{D}(a)$. Then for any $r \in F$ we have $z(x+r) \notin C_{D}(a)$, and by foregoing argument we have $\operatorname{dim}_{K} K[z(x+r)] \leq n^{\prime}$. Again, using previous lemma we obtain that $\operatorname{dim}_{K} K[x] \leq 3^{2 n^{\prime}-1}+1=n^{\prime \prime}$. Thus every element of $D$ is left algebraic over $K$ of bounded degree $n^{\prime \prime}$. Now, using Theorem 9 we are done.

Remark 1 Let $D$ be division algebra of degree $n$ over its center $F$. Also, assume that there exists element $a \in D$ such that $F(a)$ is a maximal subfield in $D$. Then for any maximal subfield $K$ of $D$ there exists $b \in D^{*}$ such that $\left\{1, b a b^{-1}, \ldots, b a^{n-1} b^{-1}\right\}$ is left independent over $K$, that is, $b a b^{-1}$ is a left algebraic element over $K$ of degree $n$ (see proof of [4, Theorem 1.3, p. 1609]).

Theorem 12 Let $D$ be a non-commutative division algebra with infinite center and $K$ a (not necessarily central) subfield of $D$. If $D^{*}$ contains a non-central normal subgroup $N$ whose elements are left algebraic over $K$ of bounded degree $n$, then $D$ is centrally finite. Furthermore, if char $(D)=0$, then $\operatorname{dim}_{F} D \leq n^{2}$.

Proof Without loss of generality assume that $K$ is a maximal subfield of $D$. If $N \subseteq K$, then $K$ is $N$-invariant and by Theorem 1 either $K=F$ or $K=D$. In both cases, $D$ is commutative, a contradiction. Hence, $N \nsubseteq \nsubseteq$. Let $a \in N \backslash K$. Since $N$ is normal in $D^{*}$ for any $x \in D^{*}$ we have $a x a^{-1} x^{-1} \in N$, which implies that $a x a^{-1} x^{-1}$ is algebraic over $K$ of bounded degree $n$. So, using previous theorem $D$ is centrally finite. Now, assume that $\operatorname{char}(D)=0$ and let $\operatorname{dim}_{F} D=l^{2}$. By [5, Theorem 7], there exists $x \in D^{*}$ such that $a x a^{-1} x^{-1}$ is algebraic over $F$ of degree $l$. Therefore, $F\left(a x a^{-1} x^{-1}\right)$ is a maximal subfield of $D$. In the view of Remark 1 , there exists $b \in D^{*}$ such that $b a x a^{-1} x^{-1} b^{-1}$ is algebraic over $K$ of degree $l$. But baxa $^{-1} x^{-1} b^{-1} \in N$ and by the assumption is algebraic over $K$ of bounded degree $n$. This implies that $l \leq n$. Thus, $\operatorname{dim}_{F} D \leq n^{2}$.

In the sequel we deal with a result in which the normal subgroup in the above theorem is the special one, the multiplicative group of commutators $D^{\prime}$. In this case we could obtain the result without additional restrictions. First we need to recall some definitions.

Let $X$ be a set of non-commuting indeterminates. For convenience, we assume that $X$ contains $m$ elements $x_{1}, \ldots, x_{m}$. Let $M$ be the free monoid generated by $X$ (it is obvious that $M$ is a subset of the Laurent polynomial $\left.\operatorname{ring} F\left[X, X^{-1}\right]\right)$. Then, $M$ is a totally ordered 
monoid with the lexicographic order $x_{1}>\cdots>x_{m}$. Every element $w=x_{i_{1}} \ldots x_{i_{t}}$ of $M$ is called a word on $X$ and $t$ is called the length of $w$. Denote by $\ell(w)=t$ the length of $w$. Let $u, v \in M$. The element $u$ is called a subword of $v$ if $v=v_{1} u v_{2}$ for some $v_{1}, v_{2} \in M$. Let $q$ be a natural number. We say that $u$ is $q$-decomposable if there exist $u_{1}, \ldots, u_{q} \in M$ such that $u=u_{1} \ldots u_{q}$ and for any non-trivial permutations $f$ of $\{1, \ldots, q\}$, one has

$$
u_{1} \ldots u_{q}>u_{f(1)} \ldots u_{f(q)} .
$$

In addition, if $(q-1) \ell\left(u_{i}\right)<\ell(u)$, then we say that $u$ is strongly $q$-decomposable.

The following lemma plays important role in our proof.

Lemma 13 [4, Theorem 2.4] Let $m, p, q$ be natural numbers and $M$ be a free monoid generated by $m$ indeterminates $X=\left\{x_{1}, \ldots, x_{m}\right\}$. Then there exists a natural number $N(m, p, q)$ such that every word $w \in M$ with $\ell(w)>N(m, p, q)$ contains either a nontrivial subword of the form $v^{p}$ or a strongly $q$-decomposable subword $u$.

Lemma 14 Let $M$ be the free monoid generated by $X=\left\{x_{1} \ldots, x_{m}\right\}$. Let $D$ be a division algebra and $K \subseteq D$ a (not necessarily central) subfield of $D$. Assume that the multiplicative group of commutators $D^{\prime}$ of $D^{*}$ is left algebraic over $K$ of bounded degree $n$. Let $N=N(m, n, n)$ be the natural number in Lemma 13. For any $w\left(x_{1}, \ldots, x_{m}\right) \in M$ and $a_{1}, \ldots, a_{m} \in D^{\prime}$, we have

$$
w\left(a_{1}, \ldots, a_{m}\right) \in \sum_{\ell\left(w_{i}\right) \leq N} K w_{i}\left(a_{1}, \ldots, a_{m}\right) .
$$

Proof For convenience, we assume that $X=\{x, y\}$ and $a_{1}=a, a_{2}=b$. The case when $X=\left\{x_{1}, \ldots, x_{m}\right\}$ is similar. Obviously, the assertion holds for every word $w(x, y)$ with $\ell(w) \leq N$. By the contrary assume that $w(x, y) \in M$ is the smallest degree lexicographic word that does not satisfy the lemma. Thus, $\ell(w)>N$ and also for any $w_{1}(x, y), \ldots, w_{t}(x, y) \in M$ with $w_{i}<w$ one has

$$
w(a, b) \notin K w_{1}(a, b)+\cdots+K w_{t}(a, b) .
$$

Now, using Lemma 13, $w$ contains either a non-trivial subword of the form $v(x, y)^{n}$ or a strongly $n$-decomposable subword $u(x, y)$.

Case 1 Assume that $w$ contains a subword $v^{n}$ and $w=v_{1} v^{n} v_{2}$ for some $v_{1}(x, y), v_{2}(x, y) \in M$. Put $c=v_{1}(a, b), d=v(a, b), e=v_{2}(a, b)$ and let $\delta_{c}: D \rightarrow$ $D$, be defined by $\delta_{c}(s)=c^{-1} s c$. Since $\delta_{c}\left(D^{\prime}\right)=D^{\prime}$, we obtain $D^{\prime}$ is left algebraic over the subfield $c^{-1} K c$ of bounded degree $n$. In particular, $d$ is left algebraic over $c^{-1} K c$ of bounded degree. Consequently, there exist $\alpha_{1}, \ldots, \alpha_{n} \in K$ such that

$$
d^{n}=c^{-1} \alpha_{1} c+c^{-1} \alpha_{2} c d+\cdots+c^{-1} \alpha_{n} c d^{n-1} .
$$

Hence,

$$
\begin{aligned}
w(a, b) & =v_{1}(a, b) v(a, b)^{n} v_{2}(a, b) \\
& =c\left(c^{-1} \alpha_{1} c+c^{-1} \alpha_{2} c d+\cdots+c^{-1} \alpha_{n} c d^{n-1}\right) e \\
& =\alpha_{1} c e+\alpha_{2} c d e+\cdots+\alpha_{n} c d^{n-1} e .
\end{aligned}
$$

For $i=1, \ldots, n$ consider $w_{i}(x, y)=v_{1}(x, y) v^{i-1}(x, y) v_{2}(x, y)$. Then Eq. (1) shows that

$$
w(a, b) \in K w_{1}(a, b)+\cdots+K w_{n}(a, b),
$$

where $w_{i}<w$ for all $i \leq n$. This contradicts relation $(*)$. 
Case 2 Assume that $w=v_{1} u v_{2}$ for some $v_{1}(x, y), v_{2}(x, y) \in M$ and strongly $n$-decomposable subword $u(x, y)$. By the definition let $u=u_{1} \ldots u_{n}$, where $u_{1}(x, y), \ldots, u_{n}(x, y) \in M$ such that $(n-1) \ell\left(u_{i}\right)<\ell(u)$ and for any non-trivial permutation $f \in S_{n}, u_{1} \ldots u_{n}>u_{f(1)} \ldots u_{f(n)}$. For any subset $T$ of $\{1, \ldots, n\}$, put $c=v_{1}(a, b), e=v_{2}(a, b)$ and $u_{T}=\sum_{i \in T} u_{i}$. By the similar argument as Case 1, $d_{T}=u_{T}(a, b)$ is left algebraic over $c^{-1} K c$ of bounded degree. Therefore, there exist $\alpha_{1, T}, \ldots, \alpha_{n, T} \in K$ such that

$$
d_{T}^{n}=c^{-1} \alpha_{1, T} c+c^{-1} \alpha_{2, T} c d_{T}+\cdots+c^{-1} \alpha_{n, T} c d_{T}^{n-1} .
$$

Note that since $(n-1) \ell\left(u_{i}\right)<\ell(u)$ we have $\ell\left(u_{i_{1}} \ldots u_{i_{k}}\right)<\ell(u)$, for all $k<n$. Thus for every $T \subseteq\{1, \ldots, n\}$ and $k<n$ one can see the length of summands of $d_{T}^{k}$ is strictly less than $\ell(u)$. Hence, for every $k \leq n$ every summand of $c d_{T}^{k} e$ is lexicographically smaller than $w$. Therefore for every $T \subseteq\{1, \ldots, n\}$, we deduce that

$$
c d_{T}^{n} e=\sum_{i=1}^{t} \beta_{i} w_{i}(a, b),
$$

where $\beta_{i} \in K$ and $w_{i} \in M$ with $w_{i}<w$.

Now, observe that

$$
u_{1} \ldots u_{n}=\sum_{f \in S_{n}, f \neq i d} u_{f(1)} \ldots u_{f(n)}-\sum_{T \subseteq\{1, \ldots, n\}}(-1)^{n-|T|} d_{T}^{n} .
$$

Hence,

$$
\begin{aligned}
w=c u e & =c u_{1} \ldots u_{n} e \\
& =\sum_{f \in S_{n}, f \neq i d} c u_{f(1)} \ldots u_{f(n)} e-\sum_{T \subseteq\{1, \ldots, n\}}(-1)^{n-|T|} c d_{T}^{n} e .
\end{aligned}
$$

Clearly, summands of the first summation are lexicographically less than $w$ and also by equation $(* *)$ the terms of the second summation above are of the form $\alpha w_{i}(a, b)$, where $w_{i}<w$. This again contradicts relation (*) and lemma is proved completely.

Before stating the proof of next result we need to recall a generalization of a theorem due to Wedderburn about spliting of a polynomial in a division algebra [8, p.250].

Theorem 15 [9, Lemma 2.1] Let $D$ be a division algebra with a maximal subfield $K$. Suppose $a \in D$ is left algebraic over $K$ and $f(t)$ is its monic minimal polynomial over $K$. Then

$$
f(t)=\left(t-x_{1} a x_{1}^{-1}\right) \ldots\left(t-x_{n} a x_{n}^{-1}\right),
$$

for some $x_{i} \in D$, and every root of $f(t)$ is conjugate to a.

From above we find that if $a \in D$ is left algebraic over a maximal subfield $K$ of degree $n$, then there are $d_{1}, \ldots, d_{n-1} \in D^{*}$ such that $a d_{1} a d_{1}^{-1} \ldots d_{n-1} a d_{n-1}^{-1} \in K^{*}$. Consequently we have

$$
a d_{1} a d_{1}^{-1} \ldots d_{n-1} a d_{n-1}^{-1}=a^{n} c,
$$

for some $c \in K[a] \cap D^{\prime}$. Therefore, we obtain that $a^{n} \in K^{*} D^{\prime}$. We state this result as following lemma.

Lemma 16 Let $D$ be a division algebra with a maximal subfield $K$. If $a \in D$ is left algebraic over $K$ of degree $n$, then $a^{n} \in K^{*} D^{\prime}$. 
Theorem 17 Let $D$ be a division algebra with center $F$ and $K$ be a subfield of $D$. Assume that every element of the multiplicative group of commutators $D^{\prime}$ of $D^{*}$ is left algebraic over $K$ of bounded degree $n$, then $\operatorname{dim}_{F} D \leq n^{2}$.

Proof Without loss of generality, we may assume that $K$ is a maximal subfield of $D$. There is nothing to do if $K=F$, so assume that $F \neq K$.

Choose $a_{1}, \ldots, a_{m} \in D^{\prime}$ arbitrarily and let $D_{m}=F\left(a_{1}, \ldots, a_{m}\right)$ denote the subdivision algebra of $D$ generated by $a_{1}, \ldots, a_{m}$ over $F$. Let $A_{m}=F\left[a_{1}, \ldots, a_{m}\right]$ be the subalgebra of $D$ generated over $F$ and $K_{m}$ be the vector space generated by $A_{m}$ over $K$. Also, let $M$ be the free monoid generated by $m$ indeterminate $X=\left\{x_{1}, \ldots, x_{m}\right\}$, and $N=N(m, n, n)$ be as in Lemma 14. By Lemma 14,

$$
K_{m}=\sum_{w \in M, \ell(w) \leq N} K w\left(a_{1}, \ldots, a_{m}\right) .
$$

Observe that the cardinality of $\left\{w\left(x_{1}, \ldots, x_{m}\right) \in M \mid \ell(w)=i\right\}$ is $m^{i}$. Thus, $K_{m}$ is a vector space of dimension $d(m) \leq 1+m+\cdots+m^{N}$ over $K$. This implies that $K^{*} D^{\prime}$ is algebraic over $K$ of bounded degree, say $t$, because $K^{*} D^{\prime}$ is a subset of $K_{m}$ and $K_{m}$ generated by $A_{m}$. Now, consider the factor group $\frac{D^{*}}{K^{*} D^{\prime}}$ which is an abelian group. By the Brauer-CartanHua Theorem we know that $D=F\left(D^{\prime}\right)$. If $d_{1}$ and $d_{2}$ are two elements of $D^{\prime}$ such that $d_{1}+d_{2} \neq 0$, then by Lemma 16 and abelian property of $\frac{D^{*}}{K^{*} D^{\prime}},\left(d_{1} d_{2}\right) K^{*} D^{\prime}$ has order at most $n$. Now, $d_{1}+d_{2}=d_{1}\left(1+d_{1}^{-1} d_{2}\right)$ implies that $d_{1}+d_{2}$ is left algebraic over $K$ of degree at most $n$. By induction, for elements $d_{1}, \ldots, d_{s} \in D^{\prime}$ with $d_{1}+\cdots+d_{s} \neq 0$, we find that $\left(d_{1}+\cdots+d_{s}\right) K^{*} D^{\prime}$ has order at most $n$ in factor group. This implies that every element of $D^{*}$ is algebraic over $K$ of degree at most $t n$. So by Theorem $9, D$ is centrally finite. Using Theorem 6, we find that $\operatorname{dim}_{F} D \leq n^{2}$. This completes the proof.

Our next result provides analogous theorem with respect to additive commutators of a division algebra.

Theorem 18 Let $D$ be a division algebra with infinite center $F$. Let $K$ be a non-central subfield of $D$ that contains an element $a \in K \backslash F$ algebraic over $F$. Assume that for every $x \in D$, ax $-x$ a is left algebraic over subfield $K$ of bounded degree $n$. Then $D$ is centrally finite.

Proof Since $a$ is non-central, there exists $b \in D$ such that $u=a b-b a \neq 0$. Thus for every $c \in C_{D}(a), a(b c)-(b c) a=u c$ is left algebraic over $K$. In particular for every $r \in F, a(b(c+r))-(b(c+r)) a=u(c+r)$ is left algebraic over $K$. Now, using Lemma 10 we find that $\operatorname{dim}_{K} K[c] \leq k+1=n^{\prime}$. In short, we proved that all elements of $C_{D}(a)$ are algebraic over $K \subseteq C_{D}(a)$ of bounded degree. Hence using Theorem 9 we obtain that $C_{D}(a)$ is centrally finite. Notice that $a$ is algebraic over $F$, so we have $\operatorname{dim}_{F} F(a)<\infty$. Hence, using Double Centralizer Theorem [8, Theorem 15.4, p. 240] we find that $Z\left(C_{D}(a)\right)=F(a)$. Therefore,

$$
\operatorname{dim}_{F} C_{D}(a)=\operatorname{dim}_{F(a)} C_{D}(a) \cdot \operatorname{dim}_{F} F(a)<\infty .
$$

Again applying Double Centralizer Theorem yields $\operatorname{dim}_{F} D<\infty$. This completes the proof.

At the end of this note we pose following conjectures. 
Conjecture 19 Let $D$ be a division algebra with center $F$ and let $K$ be a subfield (not necessarily central) of $D$. Then $D$ is left algebraic over $K$ if and only if $D^{\prime}$ is left algebraic over $K$.

Conjecture 20 Let $D$ be a division algebra with center $F$ and let $K$ be a subfield (not necessarily central) of $D$. Assume that for every $x, y \in D^{*}, x y x^{-1} y^{-1}$ is left algebraic over $K$ of bounded degree $n$. Then $\operatorname{dim}_{F} D \leq n^{2}$.

Conjecture 21 Let $D$ be a division algebra with center $F$ and let $K$ be a subfield (not necessarily central) of $D$. Then $D$ is left algebraic over $K$ if and only if $[D, D]$ is left algebraic over $K$.

Conjecture 22 Let $D$ be a division algebra with center $F$ and let $K$ be a subfield (not necessarily central) of $D$. Assume that for every $x, y \in D^{*}, x y-y x$ is left algebraic over $K$ of bounded degree $n$. Then $\operatorname{dim}_{F} D \leq n^{2}$.

Acknowledgements The research of the first author was supported by ERC grant number 320974. The second author is indebted to the Research Council of Sharif University of Technology for support. The third author acknowledges support from Vietnam National University Ho Chi Minh City (VNU-HCM) under grant number B2016-18-01.

Open Access This article is distributed under the terms of the Creative Commons Attribution 4.0 International License (http://creativecommons.org/licenses/by/4.0/), which permits unrestricted use, distribution, and reproduction in any medium, provided you give appropriate credit to the original author(s) and the source, provide a link to the Creative Commons license, and indicate if changes were made.

\section{References}

1. Albert, A.A., Muckenhoupt, B.: On mtrices of trace zeros. Mich. Math. J. 4(1), 1-3 (1957)

2. Amitsur, S.A.: Rational identities and applications to algebra and geometry. J. Algebra 3, 304-359 (1966)

3. Beidar, K.I., Martindale, W.S., Mikhalev, A.V.: Rings with Generalized Identities. Marcel Dekker, Inc., New York (1996)

4. Bell, J.P., Drensky, V., Sharifi, Y.: Shirshov's theorem and division rings that are left algebraic over a subfield. J. Pure Appl. Algebra 217, 1605-1610 (2013)

5. Chebotar, M.A., Fong, Y., Lee, P.H.: On division rings with algebraic commutators of bounded degree. Munscripta Math. 113, 153-164 (2004)

6. Herstein, I.N., Scott, W.R.: Subnormal subgroups of division rings. Canad. J. Math. 15, 80-83 (1963)

7. Jacobson, N.: Structure theory for algebraic algebras of bounded degree. Ann. of Math. (2) 46(4), 695707 (1945)

8. Lam, T.Y.: A First Course in Noncommutative Rings, 2nd edn, GTM, No. 131. Springer, New York (2001)

9. Leung, K.H.: On a generalization of Albert's Theorem. Israel J. Mth. 69(3), 337-350 (1991)

10. Mahdavi-Hezavehi, M., Akbari-Feyzaabaadi, S., Mehraabaadi, M., Hajie-Abolhassan, H.: Commutators in division rings II. Comm. Algebra 23(8), 2881-2887 (1995)

11. Rowen, L.H.: Ring Theory, Student Edition. Academic Press, Boston (1991)

12. Thompson, R.C.: Commutators in the special and general linear groups. Trans. Am. Math. Soc. 101(1), $16-33(1961)$ 